\title{
Generación de Competencias con Base en la Gestión de Conocimiento Científico
}

\author{
Generation of Competences Based on the Management of \\ Scientific Knowledge
}

\author{
José Antonio Martínez Villalba ${ }^{1 *}$ \\ Salvador Sánchez Muñoz ${ }^{2}$ \\ ${ }^{1}$ Universidad Iberoamericana Torreón, México \\ ${ }^{2}$ Universidad Politécnica de Gómez Palacio, México
}

\begin{abstract}
Actualmente se necesitan alternativas viables para mejorar el proceso de aprendizaje en los estudiantes y puedan hacer frente a las demandas de la sociedad. Generar experiencias significativas con base a proyectos de investigación de problemas reales, favorecerá que el alumnado desarrolle competencias que auxilien en la solución de los problemas que una sociedad globalizada y cambiante exige. Esta investigación busca analizar las diferentes competencias que desarrollan estudiantes en áreas relacionadas a la química y la biología a partir de la gestión del conocimiento. Para ellos se utiliza un instrumento de evaluación con 74 variables en escala de razón y 11 en escala nominal (alfa de Cronbach final de 0.950). Dicho instrumento se aplicó en dos congresos nacionales a 151 estudiantes de licenciatura hasta posdoctorado. Se realizaron análisis estadísticos para determinar la univariable y la prueba $t$ de Student con la finalidad de encontrar las principales competencias involucradas en la gestión del conocimiento. Entre los principales hallazgos se encuentran: que los estudiantes desarrollan competencias técnicas, personales e intelectuales. El área de estudio es independiente a la gestión del conocimiento. Se sugiere reforzar asignaturas relacionadas a la metodología de investigación en los programas académicos que ayuden a generar trabajos científicos, así como crear centros de investigaciones institucionales que estén vinculados con empresas.
\end{abstract}

Descriptores: Competencias del docente; Química; Biología; Gestión del conocimiento; Docente.

Currently, viable alternatives are needed to improve the learning process in students and can cope with the demands of society. Generate significant experiences based on research projects of real problems, will encourage students to develop skills that help in solving the problems that a globalized and changing society demands. This research seeks to analyze the different competences developed by students in areas related to chemistry and biology based on knowledge management. For them, an evaluation instrument with 74 variables on a ratio scale and 11 on a nominal scale (final Cronbach's alpha of 0.950) is used. This instrument was applied in two national congresses to 151 students from undergraduate to postdoctoral. Statistical analyzes were performed to determine the univariable and Student's t test in order to find the main competences involved in knowledge management. Among the main findings are: that students develop technical, personal and intellectual skills. The study area is independent of knowledge management. It is suggested to reinforce subjects related to the research methodology in the academic programs that help generate scientific works, as well as to create institutional research centers that are linked to companies.

Keywords: Teacher qualifications; Chemistry; Biology; Knowledge management; Teachers.

*Contacto: jose.martinez@iberotorreon.edu.mx

ISSN: $1696-4713$

www.rinace.net/reice/

revistas.uam.es/reice
Recibido: $1^{\text {a }}$ Evaluación: $2^{\text {a }}$ Evaluación: Aceptado:
15 de diciembre 2017

13 de enero 2018

9 de febrero 2018

2 de marzo 2018 


\section{Introducción}

Actualmente se buscan nuevas estrategias para cumplir con los retos y requisitos que exige la sociedad. Las exigencias políticas, los problemas económicos, la cultura, las presiones sociales y el conocimiento son aspectos que persistentemente permean en un proceso educativo. Así mismo, la sociedad reclama a las universidades una serie de requisitos que involucren mayor calidad educativa, modernización, eficiencia en sus egresados, responsabilidad social, equidad y una serie de habilidades, las cuales, deben de responder a los retos que involucran las necesidades de la población, exigencias del mercado laboral y tendencias en el ámbito mundial.

Estos retos involucran un cambio en el proceso educativo que permita desarrollar un aprendizaje significativo al alumnado. Se han propuesto nuevas metodologías, enfoques o herramientas para lograr dichos objetivos. Así mismo, las instituciones públicas y privadas buscan personas que tengan habilidades en el campo laboral e investigativas que demuestren conocimiento en su área de estudio. En épocas recientes, las organizaciones solicitan personas con mayor preparación académica o las empresas implementan sus propias universidades para orientar a los estudiantes recién egresados a las competencias que ellas mismas requieren con la finalidad de cumplir sus necesidades.

En los últimos años, son más las empresas que han generado sus propias universidades corporativas para hacer frente a la falta del talento certificado que egresa de las instituciones educativas. Según Gabriel Sama del diario Expansión (2013) en México se concentran el 22\% de las universidades corporativas en América Latina, sólo después de Brasil, que tiene el $34 \%$. El bajo rendimiento escolar y la falta de nuevas estrategias por parte de las instituciones educativas para aumentar las competencias o habilidades de los egresados han provocado que las empresas opten por esta opción.

México era uno de los países que más invertía en la educación en las últimas décadas a nivel mundial, sin embargo, esto dio un giro radical desde hace algunos años. Según la Organización para la Cooperación y el Desarrollo Económicos (OCDE), México es un país que gasta poco por cada estudiante, a pesar de que elevó los fondos que destina a educación del 4,4\% del Producto Interno Bruto (PIB) en el 2000, al 5,2\% en 2012, un nivel similar al promedio de otros países de la organización. De acuerdo al estudio que realizó la OCDE, se invierte alrededor de 2,600 dólares por estudiante, lejos de los 8,200 dólares del promedio de dicha organización. Además, sólo cuatro de cada 100 egresados de licenciatura acceden a un posgrado y es uno de los países con menor cantidad de profesionistas preparados de la organización, solo por arriba de Brasil, Turquía e Italia (Romero, 2016).

Por su parte, el gasto anual que se obtuvo en 2014 en el nivel superior se situó en $46 \%$ de PIB per cápita, un porcentaje arriba del promedio que maneja la OCDE (41\%). La organización infiere que este gasto se puede relacionar con el alto índice de deserción entre los estudiantes de educación media superior en México (Castañeda y Rebolledo, 2014).

Otro de los factores preocupantes es la falta de capital humano para realizar investigaciones en el país. Los mexicanos que se dedican al desarrollo de actividades científicas son apenas 200 personas por cada millón de habitantes. Así mismo, el gobierno federal invierte alrededor del $0,5 \%$ del PIB a proyectos vinculados a la ciencia y la tecnología, y aunque ha aumentado en los últimos años, no se compara con países 
como Brasil, Argentina y Estados Unidos que invierten el triple de su PIB en este rubro (Instituto de Estadística de la UNESCO, 2017).

Uno de los procesos que se pueden emplear para egresar estudiantes con alta capacidad intelectual y habilidades sociales, es la gestión del conocimiento. Dichas competencias se pueden generar mediante experiencias significativas con base en la realización de proyectos de investigación derivados de problemas reales, en los que se desarrollen habilidades que cubran la demanda de conocimientos, adaptabilidad y destrezas que requiere la sociedad y el ambiente laboral actual.

En México, una de las herramientas utilizadas para hacer frente a esta problemática, es la generación de vínculos o cuerpos académicos para crear dinámicas en la producción y gestión del conocimiento científico. Desde 2001, el gobierno federal se ha enfocado en la necesidad de formar cuerpos académicos multidisciplinarios como una solución central, en la que los profesores de tiempo completo de las universidades, puedan desarrollar investigación, otorgar asesorías, impartir docencia y preparar ponencias en torno a temas interdisciplinarios o multidisciplinares específicos que den respuestas a las necesidades humanas y de la sociedad actual (Torres y Jaimes, 2015).

La gestión del conocimiento es un término ampliamente usado a nivel industrial como estrategia de desarrollo y crecimiento para obtener mejores resultados y una economía sustentable. El consenso mundial establece que la actividad científica es el único camino para lograr el progreso de un país, y el desarrollar tecnología garantiza un avance en la sociedad y disminuye el impacto ambiental. Sin ciencia, tecnología e innovación no hay un país que pueda generar una economía sólida. Por eso, es de suma importancia generar y divulgar conocimiento científico para mejorar las circunstancias adversas en las que se encuentra el país y fomentar el desarrollo de personal competente y hábil para hacer frente a los retos que la sociedad requiere. Por esta razón, es primordial generar nuevos mecanismos para incrementar las competencias básicas, laborales e investigativas al terminar la licenciatura.

El conocimiento y las habilidades se han convertido en uno de los activos más importantes para un país. Con base en este contexto, la gestión del conocimiento se ha convertido en una herramienta esencial para mejorar el desempeño de los individuos, desarrollar habilidades, valores y competencias investigativas; además, permite la elaboración de estrategias, una dinámica de aprendizaje significativo, y el uso de metodologías y tecnologías que ayudan a la divulgación del conocimiento. Así mismo, es necesaria la gestión del conocimiento para encontrar información relevante que nos garantice tomar acciones sensatas en la resolución de problemas reales relacionados al medio ambiente, la sociedad y la tecnología. El objetivo de este trabajo fue analizar las competencias profesionales e investigativas que desarrollan los estudiantes en los cursos de química y biología a partir de los fundamentos relacionados con el proceso de la gestión del conocimiento y la gestión de proyectos, en diferentes universidades de la República Mexicana.

\section{Fundamentación teórica}

\subsection{Conocimiento y competencias educativas}

El conocimiento es considerado como el conjunto de habilidades con los cuales los individuos suelen solucionar problemas, comprender las reglas cotidianas al igual que las 
instrucciones para ejercer una acción, incluye tanto la teoría como la práctica, está ligado a las personas y se basa en datos e información; forma parte integral en cada individuo y representa las creencias de éstos acerca de las relaciones causales (Pereira, 2011).

El conocimiento es el entendimiento adquirido de algo que fue significativo. La inteligencia y la razón natural, en una sociedad, se producen cuando los individuos hacen uso de lo que saben, aprehenden de la realidad o de una relación entre objetos, y así, la información generada con base en los conocimientos puede ser manipulada para la solución de problemas que exige en forma urgente la sociedad y el mundo exterior (Bustelo y Amarilla, 2001). No hay duda de que está relacionado con otras creencias, como las opiniones, las actitudes o la ideología, entre otras.

Por otra parte, el Centro Interuniversitario de Desarrollo (2004) define a la competencia como una capacidad o habilidad individual que se desarrolla para realizar un conjunto de operaciones y tareas con base en regulaciones o normas de calidad. Se pueden distinguir varios tipos de competencias en la literatura. Por ejemplo, aquellas competencias relacionadas al conocimiento general del individuo (saber conocer), competencias técnicas (saber hacer), competencias sociales (saber convivir), competencias afines a las actitudes personales (saber ser) (Tobón, Pimienta y García, 2010) y las relacionadas a la participación (saber estar) (Santoyo, 2005).

Las competencias constituyen uno de los principales puntos de interés en nuestro sistema educativo, debido a que están orientadas al desarrollo de habilidades y destrezas para una rápida adaptación del individuo a la sociedad. Así, las instituciones educativas deben de forjar una combinación de conocimientos, capacidades y actitudes en el estudiante que se encuentra en un contexto determinado; esto con el fin de su realización, desarrollo personal, inclusión social y preparación al mundo laboral (Gómez, Miralles y Molina, 2015). Así mismo, en el contexto mundial la visión integral de las competencias es una aportación por parte del profesionista al integrar los valores, la cultura y la ética, que permitan formar criterios que contribuyan a la aplicación de sus habilidades y conocimientos de manera responsable contribuyendo a un bien común (Ortiz, Vicedo y García, 2016).

Inclusive, algunos autores hablan sobre competencias laborales; las cuales, se deben desarrollar en un ambiente específico que incluyen una gran variedad de contextos, que varían en su rutina diaria y en su mayoría se presentan en eventos complejos (Gil, 2007). Estas competencias, son las habilidades que los empleadores buscan que tengan desarrollados los recién egresados para cumplir las expectativas de las empresas o instituciones y cubrir las demandas exigentes de la sociedad.

\subsection{Gestión del conocimiento}

Las transformaciones que ha sufrido la sociedad en los últimos años han sido de una envergadura total. Con el fenómeno de la globalización, la aparición y potencialización de las nuevas tecnologías de la información y el conocimiento, se necesitan más especialistas en temas específicos, pero también se exige un conocimiento cosmopolita en su área de trabajo.

La gestión del conocimiento se ha convertido en un punto crítico e indispensable para el desarrollo social y económico. Este ha adquirido un nuevo valor de producción a nivel industrial, ya que a partir de él se llevan a cabo acciones derivadas de la combinación de la información, experiencias, valores y normas internas (Romero, 2007). Por eso, en el 
ámbito empresarial surgió la conciencia sobre la gestión del conocimiento, puesto que este se convierte en un activo de capital humano desde el momento en que el personal hace explícito su conocimiento para la organización y a su vez hace posible, mediante la infraestructura y las mediaciones en la comunicación, que el conocimiento generado adquiera sentido para la empresa o institución. La gestión del conocimiento permite crear, transformar, implementar y usar el conocimiento para solucionar problemas en un área específica (Gómez y López 2007).

Hoy en día, las instituciones buscan aumentar la competitividad, la capacidad de respuesta e innovación en los adelantos científicos. Para que la gestión del conocimiento pueda coincidir con las necesidades concretas de las organizaciones, las universidades fundadoras del conocimiento deben de comenzar a aplicar o fortalecer este concepto en procesos de docencia e investigación dentro de sus programas educativos (García y Gómez, 2015). Para que todo esto tenga un valor agregado y pueda ser utilizado, debe ser un conocimiento explícito, ya que tiene que ser cedido y pueda crear un nuevo conocimiento que será capturado para su aplicación (López, 2011). Es decir, será necesario mantenerlo activo para que no se vuelva obsoleto, se potencialice y sea cada vez más extenso y útil, pero solo los métodos de enseñanza estarán cambiando constantemente por la innovación docente (Mirabal, 2015).

Por lo tanto, dada la necesidad de las instituciones de elevar la competitividad y su capacidad de respuesta en temas científicos y de innovación, el conocimiento tiene como objetivo innovar, por lo que las instituciones empiezan a fijar nuevos modelos de gestión del conocimiento que incidan en la docencia e investigación (Christer y Acevedo, 2015). De esta manera, el conocimiento servirá de guía para la acción de las personas, para aumentar el capital intelectual de las instituciones y para enfrentar los retos de la sociedad globalizada (Torres, Cruz y Hernández, 2014).

\subsection{Cuerpos académicos}

Se puede definir como cuerpo académico a un grupo en el que sus integrantes comparten un conjunto de objetivos y metas académicas; en dichas dimensiones, se encuentran la investigación, la docencia y la extensión universitaria, y pertenecen a un campo profesional ya sea una disciplina o asignatura en una institución (Clark, 1998). Es decir, es un conjunto de profesores que les gusta y tienen herramientas para desarrollar la investigación y comparten una o más líneas afines, cuyos objetivos y metas están destinados a la generación de nuevos conocimientos a través de su alto grado de especialización, y también ejercen la docencia para lograr una educación de buena calidad.

Por lo tanto, la formación de cuerpos académicos es el medio que permite a las universidades del país alcanzar los niveles de competitividad del personal docente y de esta manera obtener la generación de conocimiento y habilidades que la sociedad está demandando (Ramírez y Herrera, 2010).

Cuando se habla de las capacidades que debe desarrollar el cuerpo académico, se refiere a las habilidades que deben adquirir o desarrollar los integrantes para desempeñar funciones de investigación, resolver problemas y tener un liderazgo científico para alcanzar los objetivos planteados desde un inicio en el trabajo investigativo. Esta parte es fundamental, porque requiere atención por parte de todo el cuerpo académico, ya que dominar las habilidades metodológicas y conceptuales ayuda a aportar una filosofía y 
característica científica particular al proceso de investigación. Entonces, perfeccionar estrategias metodológicas permite la creación e implementación de protocolos de investigación adecuados para una correcta investigación, difusión de resultados, implementación y diseminación de teorías basadas en la evidencia y de su propia evaluación (Santoyo, 2005).

Estas competencias, permiten un análisis reflexivo y crítico de los conceptos teóricos a desarrollar en una investigación. Van a permitir al cuerpo académico emplear pasos y protocolos para solventar problemas cuyas soluciones se hallan en la aplicación del método científico. De igual forma, los integrantes pueden desarrollar algunas capacidades como la de investigación, criticidad, análisis y las ya mencionadas conceptuales y metodológicas para argumentar, con sustento, las mejores alternativas a la hora de resolver problemas, etc. Desde el punto de vista de Chainé (2012), al desarrollar estas habilidades podemos gestionar proyectos eficientes y con resultados. Por último, es importante considerar la capacidad de convocatoria a otros miembros de la comunidad universitaria para participar en el desarrollo de cuerpos académicos multidisciplinarios, con la convicción de garantizar el fortalecimiento de los programas educativos, aumentar el prestigio de la institución, formar recursos humanos y propiciar ambientes académicos con gran riqueza intelectual (Grimaldo, Habib y Alfaro, 2015); así como realzar la importancia de los proyectos de investigación científica como promotores en la adquisición de aprendizajes significativos en los estudiantes.

\section{Método}

El presente estudio se hizo con base en un enfoque cuantitativo, descriptivo de poblaciones y correlacional mediante un muestreo no probabilístico (Hernández, Fernández y Baptista, 2008). Dicha investigación está enfocada en analizar las competencias que se pueden generar mediante experiencias significativas, al participar en proyectos de investigación de problemas reales en áreas relacionadas a química y biología.

\subsection{Participantes}

El perfil de la aplicación del instrumento de evaluación, este abarcó a estudiantes que participan en grupos de investigación multidisciplinarios, en áreas específicas a química y la biología a nivel nacional (México). Se consideró solo a los estudiantes que realizan investigación en las ciencias mencionadas anteriormente, tanto de escuelas públicas y privadas, como de licenciatura hasta posdoctorado.

Se encuestó a 151 estudiantes, de los cuales 88 fueron de licenciatura, 43 de maestría, 16 de doctorado y solo cuatro estudiaban una estancia posdoctoral. La mayoría se encontraban adscritos a escuelas públicas (113) y el resto (38) a instituciones privadas.

El muestreo se realizó de manera intencional o selectiva. El caso más frecuente de este procedimiento es obtener una muestra de los individuos a los que se tiene fácil acceso (Selltiz et al., 1974). De este modo, se escogieron estudiantes que participaron en dos congresos nacionales de dichas disciplinas, así como en facultades o escuelas donde se dio acceso para aplicar las encuestas por vía electrónica.

No se tomaron en cuenta los centros de investigación del país. Tampoco a grupos de investigación donde solo participan investigadores o profesores. También se descartó a 
estudiantes mexicanos que se encuentran realizando una estancia de investigación fuera del país. El último criterio fue no seleccionar grupos de investigación que pertenezcan al Programa de Mejoramiento del Profesorado (PROMEP).

\subsection{Instrumento}

Para efectos de esta investigación, se escogieron solo dos variables de medición en el instrumento, las cuales se tomaron como escala de medición: la escala nominal y la de razón, según lo establecido por Hernández, Fernández y Baptista (2008).

En la escala nominal se presentan, en algunos casos, dos o más categorías para medir la variable deseada. No presentan orden ni jerarquía y solo muestran diferencias entre las variables. En la variable "género", la persona tiene dos categorías para contestar: femenino o masculino. Para la variable "nivel de estudios", el encuestado tiene cuatro categorías para contestar: licenciatura, maestría, doctorado o estancia posdoctoral. Otros ítems que presentan categorías en el instrumento son: 1) participación en congresos, que contienen las categorías: "sí" y "no", y 2) publicación de artículos científicos, que también tienen las categorías anteriores. Se presentan algunas variables sin categorías, ya que deben ser contestadas según las características del estudiante seleccionado de manera no probabilística: por ejemplo, universidad, semestre, carrera, ciudad y edad.

En la escala de razón, se emplea un intervalo del o al 100 según la constancia con la que realicen la cuestión a investigar. Donde el o indica la ausencia del aspecto que se está evaluando y el 100 es el valor máximo que se puede otorgar. Con el fin de llegar al objetivo planteado, las 74 variables en escala de razón se dividieron en 3 secciones en el instrumento de medición. La primera sección fue orientada al desempeño académico e incluyó 17 ítems. Estas variables tienen la finalidad de analizar aquellas habilidades de percepción, construcción metodológica, meta-cognitivas, instrumentales y de pensamiento (Padilla-Canales et al., 2016) que reflejen el nivel académico del entrevistado. En la segunda sección se abordaron 18 cuestiones relacionadas al proceso de investigación. Se diseñaron ítems para medir la resolución de problemas, planeación de trabajos, diseños experimentales, destreza en la operación de equipos de laboratorio, manejo de la tecnología, administración del tiempo y entendimiento de la literatura científica (Barrera, de la Rosa Rodríguez y Chang, 2017). En la última sección, se describieron las competencias, tanto transversales como específicas. Se desarrollaron 39 ítems que involucraban cuestionamientos en el saber (interpretar datos, resultados, conocimiento, criticidad, etc.) saber hacer (habilidades, destrezas en el protocolo de investigación, etc.) y el saber estar (trabajo en equipo, liderazgo, gestión de proyectos, entre otras) (Santoyo, 2005).

Las variables están presentes en 85 ítems en el instrumento de medición: 74 variables son en escala de razón de 0-100 y 11 con una escala nominal. Para finalizar, se realizó la validación de dicho instrumento con base en las tres fases estipuladas por Castejón, Santos y Palacios (2013) con el objetivo de validar el contenido de las preguntas con tres expertos en el área y la validación en la compresión y fiabilidad del cuestionario con estudiantes universitarios de tres distintas universidades de la región norte de México.

\subsection{Procedimiento}

Como paso previo, se encuestó a estudiantes investigadores que trabajan en un cuerpo académico multidisciplinario y desarrollan un trabajo de investigación en disciplinas relacionadas a la biología y la química, con el objetivo de generar la base de datos. Los 
instrumentos de medición se aplicaron directamente en dos congresos internacionales y por vía electrónica.

Enseguida, se analizó la univariable como el primer estadígrafo descriptivo. Se determinaron las medidas de tendencia central (moda, media y mediana) a los 74 ítems de escala de razón para analizar las variables que se encuentran en el límite superior e inferior. Este análisis se generó con el propósito de explicar las principales competencias de los estudiantes investigadores que no estaban dentro de la normalidad; así como las variables que no están en este rango. También se obtuvieron los niveles de frecuencia, mínimos, máximos y desviación estándar.

El segundo estadígrafo empleado fue la prueba T de Student para medir la comparación entre dos grupos con la finalidad de identificar diferencias significativas de sus medias entre ambas categorías. Se seleccionaron las variables nominales de género y área de estudio para hacer los comparativos. Para su cálculo se usó un intervalo de confianza del 95\%. Todo el procesamiento de la información fue realizado con el paquete estadístico Statistical v.10.

\section{Resultados}

El nivel de confiabilidad del instrumento obtuvo un alfa de Cronbach de 0.950067 y un alfa estandarizada de 0.964733 , lo cual, garantiza una coherencia en las respuestas y una medida de consistencia interna del instrumento de evaluación.

Los 151 estudiantes encuestados elaboraban proyectos de investigación en diferentes universidades nacionales y locales. Se entrevistaron a jóvenes investigadores de universidades privadas y públicas de los Estados de Aguascalientes, Baja California, Baja California Sur, Chihuahua, Coahuila, Durango, Guanajuato, Hidalgo, Jalisco, Michoacán, Morelos, Nuevo León, Puebla, Querétaro, San Luis Potosí, Sinaloa, Sonora, Tabasco, Tlaxcala, Veracruz, Yucatán, Zacatecas y Ciudad de México.

Los estados de Coahuila $(27,15 \%)$ y Durango $(16,55 \%)$ representaron la mayor cantidad de estudiantes encuestados. En tercer lugar, la Ciudad de México (13,24\%). Es importante mencionar que todos los encuestados ejecutan proyectos de investigación de ciencia básica en disciplinas semejantes a la biología y la química, El 58,27\% de los entrevistados tienen nivel de licenciatura, el $28,47 \%$ de maestría, el 10,59\% de doctorado y solo el 2,64\% corresponde a estancia posdoctoral. En lo que respecta al área de estudio, prácticamente la mitad realizan investigación en áreas relacionadas a la química $(50,33 \%)$ y en menor medida a la biología $(49,66 \%)$. Fueron $73(48,34 \%)$ las personas encuestadas de género femenino y $78(51,66 \%)$ de género masculino.

En primer lugar, se presenta el análisis de la univariable con la finalidad de describir las variables relacionadas a las competencias investigativas que se encuentran dentro y fuera de la normalidad. Se obtuvo una media de medias de 82,10 con una desviación estándar de 5,64.

Las variables que oscilan con un valor mayor a 87,74 (límite superior) (cuadro 1) son: "prudencia", "deducir", "perseverante" y "actuar". Lo que nos indica que son las habilidades que pueden desenvolverse satisfactoriamente dentro de un proceso de investigación. 
En el caso de la "perseverancia", se cuestionó sobre si se trabaja hasta alcanzar los objetivos planteados en la investigación, mostrando una tendencia a respuestas positivas con una mediana de 90 y un promedio de 88,07. Del mismo modo, la variable "deducir" muestra resultados similares, a la variable antes mencionada, debido a que los estudiantes tienden a cuestionarse sobre los resultados obtenidos para establecer conclusiones. Ambas variables son habilidades investigativas que son esenciales para obtener, procesar y comunicar información en un grupo de investigación. Lo que puede ayudar a mejorar las habilidades de los investigadores para gestionar el conocimiento efectivo entre comunidades epistémicas. Es decir, pone en evidencia los procesos de generación y difusión del conocimiento en el cuerpo académico, brindando herramientas a la comunidad científica para gestar procesos de transdisciplinariedad e interdisciplinariedad.

Cuadro 1. Variables presentes en el límite superior

\begin{tabular}{lcccc}
\hline & PROMEDIO & MEDIANA & MODA & FRECUENCIA \\
\hline Prudencia & 87,947 & 90 & 90 & 46 \\
Deducir & 87,980 & 90 & 100 & 47 \\
Perseverante & 88,073 & 90 & 90 & 48 \\
Actuar & 89,232 & 90 & 90 & 50 \\
\hline
\end{tabular}

Fuente: Elaboración propia a partir de los resultados de la investigación.

Siguiendo esta misma tendencia, cuando se mide la habilidad para no cometer errores en el trabajo (prudencia) y actuar responsablemente ante situaciones inesperadas en el laboratorio (actuar) son habilidades de menor grado de integración, que dependen especialmente de la naturaleza de la investigación. Ante ambas situaciones, la respuesta de los estudiantes se inclinó de manera positiva para hacer lo que creen que es correcto en un momento preciso al experimentar. Además, actúan con sensatez en las actividades que les toca realizar en el proyecto, siempre y cuando dependa de ellos mismos.

Algunos aspectos interesantes, que están dentro de la normalidad, son el trabajo en equipo, que es fundamental en los cuerpos académicos multidisciplinarios que buscan un fin específico en su campo de investigación. El implementar experimentos con base a un protocolo y seleccionar la muestra adecuada, según las necesidades de la investigación, así como, la destreza al manejar los equipos de laboratorio, son puntos críticos en la investigación, porque son parámetros que nos permiten dar lecturas exactas y precisas de los resultados experimentales.

Una característica que demostraron tener los encuestados, fue desarrollar una actitud reflexiva ante diferentes posturas teóricas cuando se analizan los resultados obtenidos. Del mismo modo, tienen iniciativa para anticiparse a necesidades futuras y se enfocan en analizar exclusivamente evidencia científica. También, mostraron facilidad para manejar diferentes técnicas experimentales y encuentran soluciones innovadoras, al no contar con los recursos suficientes para la experimentación. Otras competencias que tienen son: trabajar hasta alcanzar los objetivos planteados, muestran criticidad, analizan la información profundamente, comprenden lecturas de temas especializados, aceptan sus propias limitaciones y aprenden fácilmente temas nuevos. Estas habilidades ayudan a la construcción de conceptos, metodologías y teorías vinculadas a su proceso de investigación, y así obtener herramientas necesarias para la toma de decisiones. 
Igualmente, muestran liderazgo en el proceso, proponen ideas y estrategias que aportan soluciones reales al proyecto. Tienen la capacidad de desarrollar un pensamiento analítico, interpretar organizadores gráficos y hacer análisis estadísticos para presentar resultados. Generan ideas y se comunican fácilmente con los integrantes del cuerpo académico. Todo lo anterior, sumado a las cualidades profesionales como honestidad, humildad y responsabilidad, presentan los investigadores en las áreas antes mencionadas.

Cuadro 2. Variables presentes en el límite inferior

\begin{tabular}{lcccc}
\hline & PROMEDIO & MEDIANA & MODA & FRECUENCIA \\
\hline Disciplina & 56,907 & 70 & 90 & 26 \\
Organizar & 59,649 & 70 & 90 & 26 \\
Autonomía & 69,834 & 80 & 90 & 27 \\
Apropiar & 71,007 & 80 & 90 & 35 \\
Comunicar & 71,113 & 80 & 90 & 28 \\
Área de Conocimiento & 73,404 & 80 & 90 & 28 \\
Diseñar & 76,146 & 80 & 80 & 33 \\
Gestionar & 76,424 & 80 & 90 & 30 \\
\hline
\end{tabular}

Fuente: Elaboración propia a partir de los resultados de la investigación.

En contraparte, el cuadro 2 muestra las variables que quedaron en el límite inferior y fueron en las que los estudiantes pusieron poco énfasis durante el proceso de investigación. La "disciplina" fue la que más alejada estuvo de la normalidad, lo que demuestra que continuamente modifican las reglas en las actividades o protocolos planteados, lo que probablemente se debe a las circunstancias de las técnicas o los distintos resultados obtenidos en la experimentación a los que se tenían planteados con anterioridad, lo que los lleva a modificar o cambiar los parámetros para obtener resultados confiables.

De igual manera, la comunicación en una segunda lengua está por debajo de la media, la mayoría de los estudiantes tienen problemas para comunicar sus ideas en inglés. Este es un punto importante en la gestión de proyectos, ya que la mayoría de la información actualizada no se encuentra en español y la divulgación del conocimiento es primordial en este idioma. El dominar los fundamentos en otro idioma es una habilidad instrumental para estas disciplinas y campos relacionados a su estudio.

$\mathrm{Al}$ cuestionar a los entrevistados sobre qué tanto profundizan en su área de estudio al participar en actividades extracurriculares, la mayoría aceptó no hacerlo frecuentemente. Esto es una limitante cuando se trabaja en disciplinas relacionadas a la química y la biología, porque son ciencias centrales que son afines a otras. Uno de los aspectos trascendentales en el proceso de investigación es participar en eventos académicos para fomentar el trabajo como un principio de interdisciplinariedad, lo que permite a los investigadores relacionarse con personas de diferentes campos de estudio. Lo anterior tiene como objetivo no difundir información aislada, dispersa, fraccionada y cuya finalidad es dar mejores explicaciones al fenómeno estudiado. Así mismo, participar en estos eventos ayuda a la formación de cuerpos académicos facilitando la gestión del conocimiento, debido a que se comparten una o más líneas de estudio, se favorece la mejora de las habilidades y aumento del conocimiento. 
De la misma manera, son muy pocos los estudiantes que participan en debates para profundizar en aprendizajes. Esto puede referirse a que los estudiantes no dominan todas las habilidades investigativas y no se preocupan por mejorar su desempeño académico en el intercambio de puntos de vistas o mediante la participación en eventos ajenos a su universidad.

Con respecto a la capacidad de gestionar proyectos de investigación, esta variable obtuvo un promedio de 76,42 , de lo cual se infiere que los estudiantes dependen de otras personas para desarrollar una investigación. La capacidad para gestionar proyectos debe de incluir habilidades como la apropiación de conocimientos, la solución de problemas, la experimentación, la transferencia de conocimiento, la implementación y la integración de soluciones como aspectos fundamentales. Como estudiantes, son difíciles de conseguir en un tiempo corto. Sin embargo, todos estos aspectos se requieren para la integración del conocimiento, la transferencia de tecnologías y el desarrollo de acciones para una efectiva gestión del conocimiento. En este caso, los entrevistados no se sienten capaces de poder gestionar un proceso de investigación de manera independiente.

Finalmente, en el límite inferior se encontró la variable de "diseñar experimentos". Este punto permite explicar la escasa experiencia que como estudiantes de ciencias naturales tienen para elaborar o adaptar diseños experimentales. Es una de las habilidades de construcción conceptual que necesitan los estudiantes, ya que les permite interpretar, describir, representar y predecir lo que sucede en un experimento, todo esto con la finalidad de dar conclusiones parciales.

Como parte de la investigación se aplicó también la prueba T de Student para demostrar si los estudiantes que trabajan en las áreas relacionadas a la química muestran mayores competencias que los estudiantes de las áreas biológicas (cuadro 3). Solo cuatro variables mostraron un nivel significativo. Estadísticamente, el valor de $t$ indica que los estudiantes que trabajan en disciplinas relacionadas con la biología se comprometen para mejorar académicamente a lo largo del proceso investigativo y así mismo, referencian mejor los artículos consultados para dar crédito al autor, que aquellos estudiantes que atienden áreas relacionadas con la química. Del mismo modo, analizan evidencias exclusivamente científicas y entienden textos con vocabulario técnico en inglés. Con estas evidencias estadísticas, se refuta la hipótesis de que los estudiantes de química muestran mayores competencias que los estudiantes de biología.

Cuadro 3. Comparativo de estudiantes que trabajan en áreas relacionadas a química y biología

\begin{tabular}{lccc}
\hline & Promedio Biología & Promedio Química & VAlor T \\
\hline Dedicación & 89,973 & 84,853 & 2,257 \\
Referenciar & 91,666 & 82,053 & 3,226 \\
Análisis de información & 86,293 & 79,320 & 2,559 \\
Inglés & 86,213 & 76,133 & 3,295 \\
\hline
\end{tabular}

Fuente: Elaboración propia con resultados de la investigación.

De la misma manera, se hizo una comparación entre hombres y mujeres para analizar los contrastes principales que presentan en el desarrollo de competencias. De las 74 variables de intervalo, solo cuatro mostraron una diferencia significativa al comparar las medias de mujeres y hombres entrevistados. Las mujeres que desarrollan un trabajo de investigación en las áreas estudiadas, motivan más a los integrantes del cuerpo 
académico que los hombres para alcanzar las metas propuestas al inicio de proyecto; así mismo, demuestran tener más paciencia y no se estresan si no les salen los resultados de manera inmediata. En contraparte, los hombres desarrollan menos estrategias para la resolución de problemas, cuando se les presentan en la práctica, en comparación con las mujeres.

\section{Discusión y conclusiones}

Con base en el objetivo planteado sobre analizar las competencias que se generan a partir de la gestión de conocimiento, los resultados presentados en este artículo muestran que, al realizar proyectos de investigación relacionados con química y biología, hombres y mujeres pueden desarrollar eficientemente competencias que algunos empleadores de instituciones públicas y privadas buscan en los recién egresados.

Los estudiantes encuestados mostraron capacidades en la toma de decisiones, liderazgo, criticidad, trabajo en equipo y, sobre todo, perseverancia para alcanzar las metas trazadas desde un inicio, entre otras. Todas estas competencias se relacionan con lo estipulado por Machado, Montes y Mena (2008), que describen a las competencias como cualquier capacidad que el individuo desarrolla en un proceso educativo con la finalidad de modelar, ejecutar (obtener, procesar, comunicar), controlar y otras de menor grado de complejidad que ayuden a solventar los problemas que se susciten. Con base en ello, se puede ayudar al alumnado de diferentes niveles educativos a que adquieran estas habilidades al gestionar conocimiento de proyectos de investigación científica. Aguado y colaboradores (2017) establecen que las competencias transversales o genéricas son las que deben de adquirir los universitarios complementariamente a sus habilidades técnicas. Estás habilidades deben incluir las interpersonales, sistémicas e instrumentales, las cuales, desde su punto de vista, son imprescindibles para desarrollar diferentes estrategias de innovación docente que ayuden a la mejora de los procesos de aprendizaje para los universitarios. Por lo que una opción viable es generar estas habilidades con base a proyectos y participación por parte de los estudiantes.

Siguiendo esta misma tendencia, la creación de cuerpos académicos multidisciplinarios ayuda a mejorar el aprovechamiento académico y el aprendizaje basado en proyectos, creando experiencias significativas que auxilien al estudiante a visualizar un contexto real de un problema que enfrenta la sociedad (López, 2010). En este mismo sentido, cuando los alumnos participan en procesos de evaluación formativa o participativa han demostrado una mayor generación de competencias, motivación y aprendizajes en comparación con la manera tradicional de enseñar por parte del profesorado (Hortigüela, Pérez y Abella, 2015).

González (2017) hace un análisis sobre la información que arrojó el Consejo de Acreditación de la Enseñanza de la Ingeniería, A.C. (CACEI) en ese mismo año, se describe la deficiencia en las competencias que tienen los recién egresados de las ingenierías acreditadas de las diferentes universidades del país. Destaca en su análisis que los ingenieros deben de tener algunos atributos básicos como: capacidad para identificar y resolver problemas, capacidad para analizar, aplicar y sintetizar conceptos a su campo de estudio, desarrollar y conducir una experimentación adecuada analizando e interpretando datos, dominio de idiomas, así como establecer conclusiones y capacidades personales. Lo anterior es escaso en los egresados, debido a que los planes de estudios 
son poco flexibles, hay una vinculación limitada al mundo laboral y la práctica curricular no se realiza en escenarios reales. Todos los aspectos realizados en este análisis pueden desarrollarse mediante la gestión del conocimiento científico al participar en cuerpos académicos multidisciplinarios.

Así mismo, Guerrero (2007) en su estudio demostró que la falta de investigación en las universidades no garantiza el desarrollo de las competencias genéricas que se asocian con la innovación, desarrollo y habilidades personales. El autor destaca que, trabajar sobre proyectos de investigación desde el pregrado, se fortalecen aquellas competencias relacionadas a la personalidad, así como las intelectuales, interpersonales, organizacionales y tecnológicas. Es evidente que, para que exista un desarrollo positivo de las diferentes competencias, se requiere dar oportunidades para ponerlas en práctica o promoverlas mediante técnicas pedagógicas que garanticen un aprendizaje efectivo en el alumno (García y Gairín, 2011).

Otro aspecto importante a destacar en la participación de estos proyectos que involucran la gestión del conocimiento, es la diversidad en la que el estudiante se desenvuelve. Pérez y colaboradores (2015) señalan que la región del país, el tipo de institución académica, el área de conocimiento y el proceso de incorporación, son características fundamentales para el desarrollo de trabajos científicos, y a su vez, pueden generar diferencias institucionales e intrainstitucionales en la misma región del país. Además, las relaciones económicas, políticas y culturales que conforman la entidad y sostienen la vida social, son prioridades que marcan el proceso de transformación del estudiante. De ahí radica la imparcialidad con las competencias que egresan los universitarios.

Para finalizar, es importante que las instituciones u organizaciones apoyen los proyectos de investigación y estos no se vean afectados por las políticas, procesos, personas e intereses de cada uno de ellos. Dichos proyectos se pueden diseñar con éxito, ya sea en el sector académico o industrial, para el crecimiento del estudiante a nivel personal e intelectual. Bajo este mismo paradigma, en México no tiene sentido la idea de que al estudiante se le puede enseñar y formar profesionalmente mediante la investigación.

\section{Referencias}

Aguado, D., González, A., Antúnez, M. y de Dios, T. (2017). Evaluación de competencias transversales en universitarios. Propiedades psicométricas iniciales del cuestionario de competencias transversales. REICE. Revista Iberoamericana sobre Calidad, Eficacia y Cambio en Educación, 15(2), 129-152. http://doi.org/10.15366/reice2017.15.2.007

Barrera, R. E. R., de la Rosa, H. y Chang, J. M. Z. (2017). Competencias investigativas en la educación superior. Revista Publicando, 4(10), 395-405.

Bustelo, C. y Amarilla, R. (2001). Gestión del conocimiento y gestión de la información. Boletín del Instituto Andaluz de Patrimonio Histórico, 8(34), 226-230.

Castañeda, R. y Rebolledo, C. (2014). Panorama de la educación: Indicadores de la OCDE. París: OCDE.

Castejón, F. J., Santos, M. y Palacios, A. (2013). Cuestionario sobre metodología y evaluación en formación inicial en educación física. Revista Internacional de Medicina y Ciencias de la Actividad Física y el Deporte, 12(1), 1-23. 
Centro Interuniversitario de Desarrollo. (2004). Competencias de egresados universitarios. Santiago de Chile: CINDA.

Chainé, S. M. (2012). Las habilidades metodológicas y conceptuales en el quehacer cotidiano de la ciencia del comportamiento. Revista Mexicana de Investigación en Psicología. 4(2), 126-129.

Christer, J. O. y Acevedo, J. C. (2015). El desarrollo de capacidades y la gestión del conocimiento en los potenciales de cambio y competitividad universitaria. Anagramas. Rumbos y Sentidos de la Comunicación, 14(27), 201-215. https://doi.org/10.22395/angr.v14n27a11

Clark, H. C. (1998). Formal knowledge networks. A study of Canadian experiences. Winnipeg: International Institute for Sustainable Development.

García, M. J. y Gairín, J. (2011). Los mapas de competencias: Una herramienta para mejorar la calidad de la formación universitaria. REICE. Revista Iberoamericana sobre Calidad, Eficacia y Cambio en Educación, 9(1), 84-102.

García, M. y Gómez, M. (2015). Prácticas de gestión del conocimiento en los grupos de investigación: Estudio de un caso. Revista Interamericana de Bibliotecología, 38(1), 13-25.

Gil, J. (2007). La evaluación de competencias laborales. Educación XXI, 10, 83-106.

Gómez, C. J., Miralles, P. y Molina, S. (2015). Presentación evaluación, competencias históricas y educación ciudadana. Revista de Estudios Sociales, 52, 9-13. https://doi.org/10.7440/res52.2015.01

Gómez, R. A. y López, M. A. (2007). La gestión de las redes sociales del conocimiento para el desarrollo de investigación y docencia. Mercados y Negocios, 16, 150-160.

González, J. M. (2017). Educación universitaria con enfermedad crónica. El siglo de Torreón. Recuperado de https://www.elsiglodetorreon.com.mx/noticia/1401606.contextolagunero.html

Grimaldo, B. V., Habib, L. y Alfaro, N. G. (2015). Competencias necesarias de un cuerpo académico para el fortalecimiento de un programa educativo. RIDE. Revista Iberoamericana para la Investigación y el Desarrollo Educativo, 10, 23-46.

Guerrero, M. E. (2007). Formación de habilidades para la investigación desde el pregrado. Acta Colombiana de Psicología, 10(2), 190-192.

Hernández, R., Fernández, C. y Baptista, P. (2008). Metodología de la investigación. Ciudad de México: McGraw-Hill.

Hortigüela, D., Pérez, A. y Abella, V. (2015). Perspectiva del alumnado sobre la evaluación tradicional y la evaluación formativa. Contraste de grupos en las mismas asignaturas. REICE. Revista Iberoamericana sobre Calidad, Eficacia y Cambio en Educación, 13(1), 35-48.

Instituto de Estadística de la UNESCO. (2017). Gasto en investigación y desarrollo (\% del PIB). Washington, DC: Grupo Banco Mundial.

López, S. (2010). Cuerpos académicos: Factores de integración y producción de conocimiento. Revista de la Educación Superior, 39(155), 7-25.

López, M. L. (2011). La relevancia de la gestión del conocimiento en las empresas. Revista Apuntes del CENES, 3O(51), 223-237.

Machado, E. F., Montes, N. y Mena, A. (2008). El desarrollo de habilidades investigativas como objetivo educativo en las condiciones de la universalización de la educación superior. Pedagogía Universitaria, 13(1), 156-180.

Mirabal, J. (2015). Gestión dinámica de conocimiento organizacional. Enlace. Revista Venezolana de Información, Tecnología y Conocimiento, 12(2), 55-78. 
Ortiz, M., Vicedo, A. y García, J. (2016). Competencias, ética y valores en la formación del especialista de Pediatría. Revista Habanera de Ciencias Médicas, 15(2), 247-258.

Padilla-Canales, C., Brooks-Calderón, P., Jiménez-Porras, L. D. y Torres-Salas, M. I. (2016). Dimensiones de las competencias científicas esbozadas en los programas de estudio de biología, física y química de la educación diversificada y su relación con las necesidades de desarrollo científico-tecnológico de Costa Rica. Revista Electrónica Educare, 20(1), 1-26.

Pereira, H. (2011). Implementación de la gestión del conocimiento en la empresa. Éxito Empresarial, 135, 1-6.

Pérez, L., Grediaga, R., Gil, M., Casillas, M., de Garay, A. y Pizzonia, C. (2015). Los académicos de las universidades mexicanas: Contexto, discusión conceptual y dimensiones relevantes para la investigación. Sociológica, 6(15), 1-23.

Ramírez, M. y Herrera, A. (2010). La dinámica del cambio de cuerpos académicos bajo un enfoque de benchmarking: El caso de dos instituciones de educación superior. Revista Internacional Administración Ë Finanzas, 3(1), 61-80.

Romero, C. A. (2007). Gestión del conocimiento, asesoramiento y mejora escolar. El caso de la escalera vacía. Profesorado. Revista de Currículum y Formación del Profesorado, 11(1), 1-28.

Romero, T. (25 de enero de 2016). Reprueba México en posgrados. Reforma. Recuperado de http://www.cursorenlanoticia.com.mx/?p=40089

Sama, G. (1 de diciembre de 2013). Empresas que crean su propia universidad. Revista Expansión. Recuperado de http://expansion.mx/expansion/2013/11/25/te-falta-talento-haz-tupropia-escuela?internal_source=PLAYLIST

Santoyo, C. (2005). Análisis y evaluación de habilidades metodológicas, conceptuales y profesionales en la formación del psicólogo. Ciudad de México: UNAM.

Selltiz, C., Jahoda, M., Deutsch, M. y Cook, S. W. (1974). Métodos de investigación en las relaciones sociales. Madrid: Ediciones Rialp.

Tobón, S., Pimienta, J. H. y García, J. A. (2010). Secuencias didácticas: Aprendizaje y evaluación de competencias. Ciudad de México: Pearson Educación.

Torres, M. T., Cruz, I. y Hernández, J. (2014). Gestión del conocimiento: Experiencias de instituciones académicas y hospitalarias. Revista Ciencias de la Salud, 12(2), 169-181. https://doi.org/10.12804/revsalud12.2.2014.03

Torres, S. A. y Jaimes, K. (2015). Producción de conocimiento mediado por TIC: Cuerpos académicos de tres universidades públicas estatales de México. Sinéctica, 44, 1-16.

\section{Breve CV de los autores}

\section{José Antonio Martínez Villalba}

Profesor de tiempo completo del Departamento de Ingenierías, Arquitectura y Diseño en la Universidad Iberoamericana Torreón y responsable del laboratorio de Ingeniería Ambiental y Metalografía en la misma Universidad. Maestría en Ingeniería Bioquímica con especialidad en Bioprocesos Ambientales por la Universidad Autónoma de Coahuila. Trabajo en líneas de investigación relacionadas al área de microbiología y remediación ambiental. Doctor en Educación por la Universidad Autónoma de La Laguna. Ha desarrollado investigaciones sobre la generación de cuerpos académicos multidisciplinarios, ha participado en foros, conferencias, ponencias, co-dirección de tesis 
y congresos en áreas ambientales y educativas. ORCID ID: 0000-0003-4878-3692. Email: jose.martinez@iberotorreon.edu.mx

\section{Salvador Sánchez Muñoz}

Docente de la Universidad Politécnica de Gómez Palacio, Director del grupo de investigación Bio-Act de la Universidad Politécnica de Gómez Palacio y docente con convenio de colaboración en la Universidad Iberoamericana de Torreón. Su perfil académico es Químico Farmacéutico Biólogo de la Universidad Juárez del Estado de Durango. Realizó una Maestría en Ingeniería Bioquímica en la Universidad Autónoma de Coahuila con reconocimiento del Programa Nacional de Posgrados de Calidad (PNPC) (2014). Ha participado 12 congresos de carácter nacional e internacional en México, dos de ellos con ponencia oral y cinco con póster. Tiene 2 artículos publicados en revistas indexadas, y 2 aceptados en el mismo rubro, 1 capítulo de libro y ha sido codirector de 10 tesistas de licenciatura. ORCID ID: 0000-0003-4594-6775. Email: salvador.sanchez.csr@hotmail.com 